\title{
ON PROJECTIONS OF FINITELY ADDITIVE MEASURES
}

\author{
THOMAS JECH ${ }^{1}$ AND KAREL PRIKRY ${ }^{2}$
}

\begin{abstract}
A theorem of Z. Frolik and M. E. Rudin states that for every two-valued measure $\mu$ on $N$, if $F: N \rightarrow N$ is such that $F_{*}(\mu)=\mu$ then $F(x)=x$ for almost all $x$. We prove that a generalization of this theorem fails for measures in general: THEOREM. There exist a translation invariant measure $\mu$ on $N$ and a function $F: N \rightarrow N$ such that $F_{*}(\mu)=\mu$, and if $A \subseteq N$ is such that $F$ is one-to-one on $A$, then $\mu(A)<\frac{1}{2}$.
\end{abstract}

Let $N=\{0,1,2, \ldots\}$ denote the set of all natural numbers. A (finitely additive) measure on $N$ is a function $\mu: \mathcal{P}(N) \rightarrow[0,1]$ which satisfies

(a) $\mu(\varnothing)=0$ and $\mu(N)=1$,

(b) if $X$ and $Y$ are disjoint subsets of $N$

$$
\text { then } \mu(X \cup Y)=\mu(X)+\mu(Y) \text {. }
$$

A measure $\mu$ is two-valued if 0 and 1 are the only values of $\mu ; \mu$ is nonprincipal if $\mu(E)=0$ for every finite set $E \subset N$.

Let $F: N \rightarrow N$ be a function. If $\mu$ is a measure on $N$, then $\nu=F_{*}(\mu)$ (the projection of $\mu$ by $F$ ) is the measure defined by

$$
\nu(X)=\mu\left(F_{-1}(X)\right) .
$$

The purpose of this paper is to prove the following

THEOREM. There exists a translation invariant measure $\mu$ on $N$ and a function $F: N \rightarrow N$ such that

(a) $F_{*}(\mu)=\mu$;

(b) if $A \subseteq N$ is such that $F$ is one-to-one on $A$, then $\mu(A) \leqslant \frac{1}{2}$.

This theorem contrasts with the following theorem of Z. Frolik and M. E. Rudin (see [1] and [2]):

THEOREM. If $\mu$ is a two-valued measure on $N$ and if $F: N \rightarrow N$ is such that $F_{*}(\mu)=\mu$, then $F(x)=x$ on a set of measure 1 .

To prove our theorem, we shall first find the measure $\mu$.

If $X$ is a set of natural numbers, and $k \in N$, we let

Received by the editors September 13, 1977 and, in revised form, April 24, 1978.

AMS (MOS) subject classifications (1970). Primary 54D35; Secondary 04A20, 28A60, 54H10.

Key words and phrases. Finitely additive measure, two-valued measure, ultrafilter, projection, ultrafilter limit, integral.

${ }^{1}$ Research partially supported by NSF grant MSP 76-05993.

${ }^{2}$ Research partially supported by NSF grant MCS 74-06705-A03. 


$$
X+k=\{n+k: n \in X\}, \quad k X=\{k \cdot n: n \in X\} .
$$

Let $\left\{a_{n}: n \in N\right\}$ be a bounded sequence of real numbers, and let $\nu$ be a two-valued measure on $N$. Then there exists a unique real number $a$, which we denote

$$
a=\lim _{\nu} a_{n}
$$

such that for every $\varepsilon>0$ there exists $X$ with $\nu(X)=1$ such that $\left|a-a_{n}\right|<\varepsilon$ whenever $n \in X$. If $\nu$ is nonprincipal then $a$ is an accumulation point of the sequence $\left\{a_{n}: n \in N\right\}$.

LEMMA. There exists a measure $\mu$ on $N$ such that for every $X \subseteq N$,

(a) $\mu(X+1)=\mu(X)$, and

(b) $\mu(2 X)=\frac{1}{2} \mu(X)$.

Proof. For each $X \subseteq N$ and each $n \in N$, let

$$
X(n)=\text { the number of elements of } X \cap\{0, \ldots, n-1\} .
$$

As a first step, we pick an arbitrary nonprincipal two-valued measure $\nu$ and define, for each $X \subseteq N$,

$$
\mu_{0}(X)=\lim _{\nu} X(n) / n .
$$

It is easy to verify that $\mu_{0}$ is a nonprincipal measure on $N$, that $\mu_{0}$ satisfies

$$
\mu_{0}(X+1)=\mu_{0}(X)
$$

for all $X$, and that for every $k \in N$,

$$
\mu_{0}(k N)=1 / k \text {. }
$$

Next we consider a sequence of measures $\mu_{n}, n \geqslant 1$, defined as follows:

$$
\mu_{n}(X)=\frac{1}{n} \cdot \sum_{k=0}^{n-1} 2^{k} \cdot \mu_{0}\left(2^{k} X\right) \text {. }
$$

It is clear that each $\mu_{n}$ is a nonprincipal measure on $N$ : the only property not immediately clear is $\mu_{n}(N)=1$, but that follows from (7). Also, since $\mu_{0}$ satisfies (6), each $\mu_{n}$ satisfies

$$
\mu_{n}(X+1)=\mu_{n}(X)
$$

for all $X \subseteq N$.

Now we define, for all $X \subseteq N$,

$$
\mu(X)=\lim _{\nu} \mu_{n}(X) .
$$

Again, $\mu$ is a nonprincipal measure on $N$, and

$$
\mu(X+1)=\mu(X)
$$

for all $X \subseteq N$. We will complete the proof of Lemma by showing that for all $X$,

$$
\mu(2 X)=\frac{1}{2} \mu(X)
$$


Let $X \subseteq N$ be arbitrary. For each $n \geqslant 1$ we have

$$
\begin{aligned}
\left|\mu_{0}(X)-2 \mu_{n}(2 X)\right| & =\left|\frac{1}{n} \cdot \sum_{k=0}^{n-1} 2^{k} \mu_{0}\left(2^{k} X\right)-\frac{2}{n} \sum_{k=0}^{n-1} 2^{k} \mu_{0}\left(2^{k+1} X\right)\right| \\
& =\frac{1}{n} \cdot\left|\mu_{0}(X)-2^{n} \mu_{0}\left(2^{n} X\right)\right| \leqslant \frac{1}{n}
\end{aligned}
$$

because $\mu_{0}(X) \leqslant 1$ and $\mu_{0}\left(2^{n} X\right) \leqslant \mu_{0}\left(2^{n} N\right)=1 / 2^{n}$ by (7).

Applying $\lim _{\nu}$ to both sides of (13), we get $|\mu(X)-2 \mu(2 X)|=0$ and (12) follows.

Proof of Theorem. Let us define the function $F$. Each natural number $m>0$ can be written uniquely as

$$
m=2^{n} \cdot(2 k+1) \text {. }
$$

Let $F(m)$ be the unique $k$ such that (14) holds (and $F(0)=0$ ). The function $F$ satisfies

$$
F(2 k+1)=k
$$

and, for all $m \in N$,

$$
F(2 m)=F(m)
$$

We will show that $F$ has the properties (a) and (b) in the Theorem.

For each $n \in N$, let

$$
A_{n}=2^{n}(2 N+1) \text {. }
$$

The sets $A_{n}$ are pairwise disjoint, and for each $n, \mu\left(A_{n}\right)=1 / 2^{n+1}$. Let $B_{n}=\cup_{k=0}^{n} A_{k}$; hence $\mu\left(B_{n}\right)=1-1 / 2^{n+1}$.

To prove the property (a) of the Theorem, let $X \subseteq N$; we will show that $\mu\left(F_{-1}(X)\right)=\mu(X)$.

It follows from the definition of $F$ that for every $n \in N$,

$$
f_{-1}(X) \cap A_{n}=2^{n}(2 X+1) \text {. }
$$

Consequently, if we denote $a=\mu(X)$, and if $n \in N$, then

$$
\mu\left(f_{-1}(X) \cap B_{n}\right)=a \cdot\left(1-1 / 2^{n+1}\right)
$$

and

$$
\mu\left(B_{n}-f_{-1}(X)\right)=(1-a)\left(1-1 / 2^{n+1}\right) .
$$

Now if $n$ tends to infinity, (19) and (20) lead to $\mu\left(f_{-1}(X)\right)=a$, which proves the claim.

To prove the property (b) of the Theorem, let $X \subseteq N$ be such that $F(x) \neq F(y)$ whenever $x, y \in X$ and $x \neq y$; we will show that $\mu(X) \leqslant \frac{1}{2}$.

Let $a=\mu(X)$ and suppose, without loss of generality, that $0 \notin X$. From (16) and because $F$ is one-to-one on $X$ it follows that the sets

$$
X, 2 X, 4 X, \ldots, 2^{n} X, \ldots
$$


are mutually disjoint. Moreover, $\mu(X)=a, \mu(2 X)=a / 2, \ldots, \mu\left(2^{n} X\right)=$ $a / 2^{n}$, etc. Hence if $a>\frac{1}{2}$, then there is $n$ such that $a+a / 2+\cdots+a / 2^{n}$ $>1$ and since the sets (21) are disjoint, we have

$$
\mu\left(X \cup \cdots \cup 2^{n} X\right)=a+a / 2+\cdots+a / 2^{n}>1,
$$

a contradiction.

The proof of the Theorem is thus complete.

REMARKS. 1. The measure $\mu$ constructed above has the property that for each $X \subseteq N$,

$$
\lim \inf \frac{X(n)}{n} \leqslant \mu(X) \leqslant \lim \sup \frac{X(n)}{n} .
$$

(One proves first, by induction on $k$, that each $\mu_{k}$ has this property, using the fact that

$$
\lim \sup \frac{(2 X)(n)}{n} \leqslant \frac{1}{2} \lim \sup \frac{X(n)}{n},
$$

and similarly for lim inf.)

A set $X \subseteq N$ is said to have a density if the limit $d(X)=\lim X(n) / n$ exists. Thus if $X \subseteq N$ has a density, then $\mu(X)=d(X)$.

2. By a modification of the proof of Lemma, one can construct a measure on $N$ such that for every $X \subseteq N$,

(a) $\mu(X+1)=\mu(X)$, and

(b) $\mu(k X)=\mu(X) / k$, for all $k \geqslant 1$.

This enables one to improve " $\mu(A) \leqslant \frac{1}{2}$ ", in part (b) of the Theorem, to " $\mu(A)<\varepsilon$ ". K. Kunen and M. Yasumoto have improved this to " $\mu(A)=0$ ".

The following remarks were made by the referee. In set theory without the axiom of choice, assuming the existence of a nonprincipal ultrafilter is known to be strictly stronger than assuming the existence of a nonprincipal measure; see [3]. The results in this paper require only the assumption that there is a nonprincipal measure on $N$. Note that the "ultrafilter limit" of a bounded sequence of real numbers is really the integral with respect to the measure $\nu$, and all the constructions in this paper work just as well if $\nu$ is any nonprincipal real-valued measure. The measure $\mu$ defined by formulas (8) and (10) could be described more simply as

$$
\mu(X)=\int 2^{m} \cdot \mu_{0}\left(2^{m} X\right) d \mu_{0}(m) .
$$

Since $\mu_{0}(X)=\mu_{0}(X+1)$ for every $X \subseteq N$, it follows that

$$
\int f(m+1) d \mu_{0}(m)=\int f(m) d \mu_{0}(m)
$$

for every bounded function $f: N \rightarrow R$; hence 


$$
\begin{aligned}
\mu(2 X) & =\int 2^{m} \cdot \mu_{0}\left(2^{m+1} X\right) d \mu_{0}(m) \\
& =\frac{1}{2} \int 2^{m+1} \cdot \mu_{0}\left(2^{m+1} X\right) d \mu_{0}(m) \\
& =\frac{1}{2} \int 2^{m} \cdot \mu_{0}\left(2^{m} X\right) d \mu_{0}(m)=\frac{1}{2} \mu(X) .
\end{aligned}
$$

The authors wish to thank J. Anderson, F. Galvin, R. Herman and B. Yood with whom they had several valuable discussions.

\section{REFERENCES}

1. Zdenĕk Frolík, Fixed points of maps of $\beta N$, Bull. Amer. Math. Soc. 74 (1968), 187-191.

2. Mary Ellen Rudin, Partial orders on the types in $\beta N$, Trans. Amer. Math. Soc. 155 (1971), 353-362.

3. David Pincus and Robert M. Solovay, Definability of measures and ultrafilters, J. Symbolic Logic 42 (1977), 179-190.

Department of Mathematics, Pennsylvania State University, McAllister Bullding, University Park, Pennsylvania 16802

Department of Mathematics, University of Minnesota, Minneapolis, Minnesota 55455 